\title{
MECE Method For Categorising Typing Errors
}

\author{
Akiyo Kano \\ Child Computer Interaction Group \\ University of Central Lancashire \\ Preston \\ United Kingdom \\ akano@uclan.ac.uk
}

\begin{abstract}
This research aims to create an MECE (Mutually Exclusive, Collectively Exhaustive) categorisation method for typing errors. The research is grounded in theory by gathering typing error types found in both $\mathrm{HCI}$ and psychology literature. Empirical studies gathering typing errors from children are used to validate these error types. It is hoped that at a later date, this categorisation method can be used to detect dyslexia in children by inspecting their typing errors.
\end{abstract}

\section{Categories and Subject Descriptors}

H.5.2 [Information Interfaces and Presentation]: User Interfaces - Input Devices and Strategies, Interaction Styles, Evaluation/Methodology.

\section{General Terms}

Classification method, typing errors.

\section{Keywords}

Typing error classification, categorising error, text input, children's typing, dyslexia.

\section{INTRODUCTION}

This research began by investigating the links between the cognitive difficulties experienced by dyslexic children, with the task typing, as typing involves many cognitive processes that dyslexics find difficult to carry out. However, as the literature review progressed, it became clear that there were not enough work carried out in studying how children type, such as general guidelines on how to run text input studies with children, or a thorough categorisation method of typing errors. In order to gain the "big picture" of how each child makes typing mistakes and how one child's pattern of typing errors differ from another child's, a method that can not only count how many errors the child makes, but what kind of errors they make is vital.

The focus of the research is now to create a set of tools to gather and analyse children's typing errors. The main tool in the set is the categorisation method that aims to identify and categorise every error made by children.

\section{RESEARCH QUESTIONS}

The research will aim to answer the following questions:

(C) The Author 2008

Published by the British Computer Society
To what extent can errors be captured and understood? Although it is possible to capture data that contains all typing errors made, is it possible to understand how they were made and categorise them? It is crucial to gather more literature regarding what error types are theoretically possible, and gather vast amount of typing error data in order to answer this question.

Can text input errors be categorised automatically? Attempts have been made to categorise some common and basic groups of errors automatically. Although $100 \%$ automated categorisation is not required for the research, a software tool that can categorise most frequent errors and highlight the ambiguous, rare ones to the investigator will reduce the analysis time and increase accuracy.

Are text input errors and learning disabilities related? Although the priority of this research is to complete the categorisation method, some initial work will be carried out in investigating if there is a link between certain characteristics or symptoms of dyslexia that causes a child to make more of certain typing error types. This will provide a foundation work for post-doctorial study to answer the question of whether there is a possibility that typing tests can be used to identify the help each child requires.

\section{MAIN CONTRIBUTIONS TO HCI}

In answering the above questions, the research also aims to provide some new contributions to the field to help other researcher studying both adults and children typing:

Review of literature relating to typing errors from 1920s to the current date - Although classification methods used in recent years have now been reviewed in [2], even earlier studies dating back to the $1920 \mathrm{~s}$ are available. A journal paper reviewing the progression of text input error analysis will be pivotal in the research to gather all theoretically possible error types, but will also be useful for other text input researchers.

To create a thorough classification method - The classification method should be complete and thorough for classifying typing errors made by both adults and children.

To create useful corpus of children's typing and typing errors - by gathering copy typing data from studies involving several schools, it is hoped that this will provide a large database of children's typing log and typing errors.

Provide guidelines and tools for researchers in text input analysis with children and adults - A new phrase set that can be used both for adults and children have already been created. Data gathering software, similar to that created by Soukoreff and MacKenzie [4] is to be created that is suitable for use with children and will also allow researchers to input questions to ask about each participants. From carrying out several largescale studies, a guideline for carrying out accurate text input experiment with children will also be published. 


\section{METHODOLOGY}

The categorisation of text input errors on typewriters has been a long running theme in $\mathrm{HCI}$ and psychology that began in the years before the computer. These literature spans from 1920s to the current date. They will be used as a framework to build the categorisation method upon, by collecting every typing error types defined in them.

Large-scale empirical studies will be used to see if the typing error types found in literature actually occur in the real world. The studies will also attempt to find any new typing errors that are so far not defined in the literature.

\section{COMPLETED WORK}

The preliminary stages of creating this categorisation method have already been carried out in the form of surveying the modern literature relating to text input error analysis and gathering and analysing typing data from children in two studies with local schools.

\subsection{Children's Phrase Set}

A new phrase set, Children's Phrase Set (CPSet) has been created specifically for use with children, and is intended to be similar to a commonly used phrase sets designed for adults called TEPS[3]. The set contains 500 phrases taken from children's books and nursery rhymes and is designed to be suitable for anyone above the age of 6 years old. The full description of the phrase set can be found in [1].

\subsection{Pilot Study}

A pilot study was carried out at a local junior school, involving 40 children between 7 and 10 years old. 22 were boys and 18 were girls. The details of the set up and procedure are described in [1].

The main findings of this study was that both TEPS and CPSet can be used interchangeably, with no significant difference between the amount of error created during copying phrases from either sets. The study also generated a small corpus of typing errors (404 errors were found), which were categorised into different error types and patterns began to emerge on how to group these types. However, it was felt that forty children were too few for the researcher to feel confident in having captured realistic proportion of typing errors made by children.

\subsection{Study 2}

A second study was carried out in another local junior school to gather more typing errors. The study involved 72 children, 37 boys and 35 girls between the age of 5 and 10 years old. It was set up in the same manner as the first pilot study, with the children carrying out exactly the same tasks. The study, together with the pilot study has gathered 2290 errors, which were analysed and counted by manual inspection. This was surprisingly time consuming by hand. It was felt that to carry out much larger studies, it is crucial to have an automated categorisation program, which would at a minimum, find all the common typing mistakes in order to reduce the amount of work that has to be done manually.

\subsection{Creating ExpECT}

The typing errors gathered in the two studies were categorised manually into groups. The process involved using predefined error types found in literature, and where none were suitable for an error, a new category was created. The error types and their definitions are described in detail in [2]. ExpECT categorisation method was established from 2290 errors found from 112 children.

In [2], ExpECT was compared with two other well-known categorisation methods in completeness of categorising all errors that were found. The ExpECT method was found to be the most complete and did not suffer from the problem of breaking more complex errors into several simpler forms of errors that Wobbrock \& Myers' method [5] suffered from. The comparison of these methods is detailed in [2].

It is acknowledged that the ExpECT categorisation method suffers from two weaknesses. First is that the categorisation method was derived only from a very practical approach to gathering typing errors from children. It lacks the theoretical approach of defining all error types that are theoretically possible, some of which are rare but still possible. It is necessary to go through as much literature as possible and gather all error types that other researchers have found and defined in their studies.

Secondly, the method is based on errors created by 112 children. For the categorisation method to be applicable to children in general, the method must be based on much larger number of children. To make this possible, again, it is clear that an automation of the collection and analysis of data is the crucial next step to the research.

\section{DISSCUSSION AND FUTURE WORK}

The new categorisation method now categorises every error that was found in the data gathered, in a thorough manner, and with relatively few ambiguous error types. However, further literature survey into even earlier literature to gather all theoretically possible error types and gathering substantial amount of data from children across the country is required for the categorisation method to be MECE (Mutually Exclusive, Collectively Exhaustive).

\section{REFERENCES}

[1] Kano, A., Read, J. C., and Dix, A. (2006), Children's Phrase Set for Text Input Method Evaluations, in Proceedings of the Fourth Nordic Conference on HumanComputer Interaction - NordiCHI. 2006, ACM Press: Oslo, Norway.

[2] Kano, A., Read, J. C., Dix, A., and MacKenzie, I. S. (2007), Expect: An Expanded Error Categorisation Method for Text Input, in HCI 2007. 2007, ACM: Lancaster, UK.

[3] MacKenzie, I. S. (2006), Teps, a Shortened Name for the Phrase Set, Personal Communication. 2006.

[4] Soukoreff, R. W. and MacKenzie, I. S. (2003), Metrics for Text Entry Research: An Evaluation of Msd and Kspc, and a New Unified Error Metric. Proceedings of the Sigchi Conference on Human Factors in Computing Systems. 2003, Ft. Lauderdale, Florida, USA: ACM Press $113-120$

[5] Wobbrock, J. O. and Myers, B. A. (2006), Analyzing the Input Stream for Character- Level Errors in Unconstrained Text Entry Evaluations. in ACM Transactions on Computer-Human Interaction (TOCHI). 2006: ACM. 